\title{
Gerenciamento do cuidado em estratégias saúde da família na percepção de enfermeiros
}

\author{
Care management in family health strategies in nurses' perception
}

Gestión de la atención en las estrategias de salud familiar en la percepción de las enfermeras

\section{Matheus Couto Bica ${ }^{\mathrm{I}}$, Luiza Cremonese ${ }^{\mathrm{II}}$, Camila Nunes Barreto ${ }^{\mathrm{III}}$, André Luiz Machado Rodrigues $^{\mathrm{IV}}$, Fernanda Quevedo Alvesv}

Resumo: Objetivo: conhecer as percepções e práticas dos enfermeiros acerca do gerenciamento do cuidado. Método: estudo qualitativo descritivo, com sete enfermeiros atuantes na atenção primária à saúde, de um município no interior do Rio Grande do Sul, Brasil. A coleta de dados ocorreu de novembro a dezembro de 2019. Utilizou-se entrevista semiestruturada e análise temática. Resultados: os enfermeiros demonstraram habilidades e competências inerentes ao gerenciamento, e formação direcionada para um atendimento pautado na integralidade. Relataram dificuldades como a manutenção da continuidade de materiais. Quanto aos recursos humanos, há uma preocupação dos profissionais em conhecer as potencialidades das equipes, a fim de direcioná-las à prestação do cuidado. O trabalho em equipe possui fragilidades, uma vez que há tarefas atribuídas que necessitam ser discutidas e compartilhadas. Conclusão: os profissionais nos diferentes níveis de gestão precisam somar esforços para proporcionar condições que visam atender ao princípio da integralidade.

Descritores: Gestão em saúde; Atenção primária à saúde; Estratégia saúde da família; Enfermagem; Serviços de saúde

Abstract: Objective: to know the nurses' perceptions and practices of care management. Method: descriptive, qualitative study, with seven nurses working in primary health care, from a city in inland Rio Grande do Sul, Brazil.

\footnotetext{
${ }^{\text {I }}$ Enfermeiro, Coordenador de Enfermagem e Atendimento Domiciliar em Unimed Centro/RS. Pós-graduando em Gestão em Serviços de Saúde. Cachoeira do Sul, Rio Grande do Sul, Brasil. E-mail: matheuscoutobica@gmail.com. ORCID: https://orcid.org/0000-0003-2767-8447

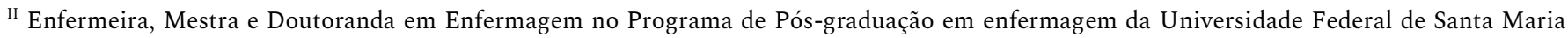
(PPGEnf/UfSM). Professora de Enfermagem na Universidade Luterana do Brasil (ULBRA) Campus Cachoeira do Sul. Cachoeira do Sul, Rio Grande do Sul, Brasil. E-mail: lu_cremonese@hotmail.com ORCID: https:/orcid.org/0000-0001-7169-1644

III Enfermeira, Mestra e Doutoranda em Enfermagem no PPGEnf/UFSM. Professora de Enfermagem na ULBRA Camous Cachoeira do Sul. Trabalha na Secretaria Estadual de Saúde do Rio Grande do Sul. Lotada na 8 a Coordenadoria Regional de Saúde de Cachoeira do Sul. Cachoeira do Sul, Rio Grande do Sul, Brasil. E-mail: camilabarreto_6@msn.com ORCID: https://orcid.org/0000-0001-5638-550X

IV Acadêmico de Enfermagem na ULBRA Campus Cachoeira do Sul. Cachoeira do Sul, Rio Grande do Sul, Brasil. E-mail: andre.machadoro@gmail.com ORCID: https://orcid.org/0000-0002-3197-804X

v Enfermeira, Mestra em Promoção da Saúde pelo Programa de Pós-graduação em Promoção da Saúde da Universidade de Santa Cruz do Sul (PPGPS/UNISC). Professora e Coordenadora de Enfermagem na ULBRA Campus Cachoeira do Sul. Cachoeira do Sul, Rio Grande do Sul, Brasil. Email: feqalves@yahoo.com.br ORCID: https://orcid.org/0000-0003-4450-0608
} 
Gerenciamento do cuidado em estratégias saúde da família na percepção de enfermeiros $\mid 2$

Data were collected from November to December 2019. Semi-structured interviews and thematic analysis were used. Results: nurses demonstrated skills and competencies inherent to management, and training directed to an integrality-based care. They reported difficulties such as the maintenance of the continuity of materials. As for human resources, there is a concern of professionals to know the potential of the teams, in order to direct them to the provision of care. Teamwork has weaknesses, since there are assigned tasks that need to be discussed and shared. Conclusion: professionals at different levels of management need to join forces to provide conditions that aim to meet the principle of integrality.

Descriptors: Health management; Primary health care; Family health strategy; Nursing; Health services

Resumen: Objetivo: conocer las percepciones y prácticas de las enfermeras sobre la gestión de la atención. Método: estudio cualitativo descriptivo, con siete enfermeras trabajando en atención primaria de salud, de un municipio en el interior de Rio Grande do Sul, Brasil. Los datos se recopilaron de noviembre a diciembre de 2019. Se utilizaron entrevistas semiestructuradas y análisis temáticos. Resultados: las enfermeras demostraron habilidades y competencias inherentes a la gestión, y la formación dirigida a la atención basada en la integralidad. Informaron dificultades como el mantenimiento de la continuidad de los materiales. En cuanto a los recursos humanos, existe la preocupación de los profesionales por conocer el potencial de los equipos, con el fin de dirigirlos a la prestación de atención. El trabajo en equipo tiene debilidades, ya que hay tareas asignadas que deben ser discutidas y compartidas. Conclusión: los profesionales de los diferentes niveles de gestión deben unir fuerzas para proporcionar condiciones que tienen como objetivo cumplir con el principio de integralidad.

Resumen: Gestión en salud; Atención primaria de salud; Estrategia de salud familiar; Enfermería; Servicios de salud

\section{Introdução}

A Atenção Primária à Saúde (APS) é um nível organizacional do atendimento de saúde que possibilita atender a maior parte das necessidades de uma população de maneira regionalizada, contínua e sistematizada. ${ }^{1}$ A Estratégia Saúde da Família (ESF) viabiliza o acesso do usuário ao Sistema Único de Saúde (SUS), consolida as propostas de ações da Atenção Básica e estimula mudanças nas relações de trabalho estabelecidas entre os profissionais da saúde e os usuários. Além disso, promove a reorganização do modelo de atenção à saúde. ${ }^{1}$ Nesse contexto, destaca-se o enfermeiro que, ao desenvolver suas atividades diárias, trabalha de maneira próxima com outros profissionais da área da saúde e com atores sociais.

Dentre as atividades do enfermeiro, destaca-se o gerenciamento do cuidado. Esse conceito é entendido como uma atribuição do enfermeiro diretamente relacionada à busca pela qualidade assistencial e por melhores condições de trabalho para os profissionais, a partir de 
uma perspectiva que articula gerência e assistência, tendo como foco o usuário do serviço de saúde e o cuidado em uma abordagem que supera o tecnicismo em direção à integralidade do cuidado. ${ }^{2}$

A gestão ou o gerenciamento do cuidado são aplicados à articulação das dimensões gerencial e assistencial no processo de trabalho. Quando o enfermeiro atua na dimensão gerencial, ele desenvolve ações voltadas à organização do trabalho e de recursos humanos, cujo propósito é viabilizar as condições adequadas tanto para a oferta do cuidado ao paciente quanto para a atuação da equipe de enfermagem. Já a dimensão assistencial define como foco de intervenção as necessidades do cuidado de saúde com a finalidade de atendê-las em um formato integral. ${ }^{3}$

Nessa perspectiva, a integralidade do cuidado pressupõe ações de saúde que considerem as dimensões gerencial e assistencial. A integralidade é permeada pela priorização de ações de promoção e prevenção, atenção nos diferentes níveis de complexidade em saúde, articulação das implementações de melhorias, proteção, diligência e abordagem integral do indivíduo e das famílias. A integralidade perpassa a necessidade de repensar práticas e conformações dos serviços públicos de saúde que ainda hoje são caracterizados pela descontinuidade assistencial, o que fragiliza o atendimento prestado à população. ${ }^{4}$

Destacam-se como atividades gerenciais do Enfermeiro na ESF: a participação na elaboração, coordenação e articulação do planejamento da Unidade de Saúde (US); promoção da integração e do bom relacionamento das equipes de saúde sob sua supervisão; investimento nas relações interpessoais (valorizando o desempenho dos profissionais); identificação dos recursos físicos e do perfil demográfico e epidemiológico da população adscrita. ${ }^{1}$ Assim, os profissionais de saúde, dentro de suas competências multiprofissionais, precisam estar aptos a desenvolver ações de prevenção, promoção, proteção e reabilitação da saúde, tanto em nível individual quanto coletivo.

Compreende-se que as ações gerenciais do enfermeiro devem apresentar como finalidade a qualidade do cuidado. Espera-se que a cisão entre as dimensões cuidar e gerenciar não comprometa a qualidade e provoque conflitos no trabalho do enfermeiro, seja do profissional com a sua própria prática ou em sua relação com a equipe de enfermagem e de saúde. ${ }^{5}$ 
Gerenciamento do cuidado em estratégias saúde da família na percepção de enfermeiros $\mid 4$

A literatura apresenta um quantitativo maior de produção de conhecimento acerca do gerenciamento do cuidado no campo hospitalar, ${ }^{6}$ o que justifica a importância deste estudo. A partir disso, tem-se como questão de pesquisa: quais são as percepções e práticas dos enfermeiros acerca do gerenciamento do cuidado? Tendo como objetivo conhecer as percepções e práticas dos enfermeiros acerca do gerenciamento do cuidado.

\section{Método}

Trata-se de uma pesquisa de campo, com abordagem qualitativa e de caráter descritivo. Como critério de seleção, os participantes do estudo foram sete enfermeiros de ESF de um município no interior do Rio Grande do Sul, Brasil. O estudo foi realizado em sete unidades, sendo seis em perímetro urbano e uma em área rural. Dentre os profissionais entrevistados, cinco têm pós-graduação e desses apenas três estão ligados à saúde pública. O quantitativo de unidades e enfermeiros constitui a totalidade do município. Para a captação dos integrantes, foi realizado contato pessoal, oferecendo o Termo de Consentimento Livre e Esclarecido e a Carta de Anuência. Após o aceite e assinatura dos termos, foi realizado agendamento conforme local de preferência. Não houve recusas em participar.

Para coleta de dados, foram realizadas entrevistas semiestruturadas pelo pesquisador, em novembro e dezembro de 2019, nos locais de serviço dos entrevistados, tendo em média a duração de 27 minutos cada. Adotou-se o critério de saturação dos dados ao serem alcançados os objetivos..$^{7-8}$ As entrevistas foram gravadas e transcritas posteriormente. O roteiro de entrevista foi composto por sete perguntas, sendo elas: o que é para você o gerenciamento em Enfermagem? Como você percebe o preparo do enfermeiro para essa prática? Como é organizado o processo de cuidar em Enfermagem com sua equipe? Você já criou algum protocolo durante as práticas? Para você, como acontece a gestão da equipe? Quais as facilidades e/ou dificuldades, ou conflitos nas relações de trabalho entre você e a equipe? Você 
5 | Bica MC, Cremonese L, Barreto CN, Rodrigues ALM, Alves FQ

tem realizado capacitações/educação permanente com sua equipe?. Os depoimentos foram organizadas em sistema alfanumérico para preservar o anonimato dos participantes (E1, E2, E3...), sendo "E” para enfermeiro e a numeração conforme ordem cronológica das entrevistas. Para o tratamento do material, foi utilizada a análise de conteúdo temática. ${ }^{7}$

Cabe destacar que todos os padrões éticos da Resolução 466/12 do Conselho Nacional de Saúde - Ministério da Saúde, referente às pesquisas com seres humanos, foram seguidos. Também, foi providenciada a Carta de Anuência para autorização da Coordenação de Atenção à Saúde do Município com posterior aprovação do Comitê de Ética em Pesquisa com Seres Humanos, sob parecer n⿳0 3.678.162, em 1ํㅡㄹ de novembro de 2019 .

\section{Resultados}

Foi evidenciado a preocupação com que não faltem os materiais, uma vez que são fundamentais para que a assistência aos clientes aconteça. Emergiu a estratégia de realizar um estoque próprio dentro da unidade, uma vez que o suprimento dos materiais não mantém uma estabilidade. Ainda, para a realização de grupos, acabam precisando fazer a aquisição do material.

Em relação aos materiais, é tu teres o controle do que tem, o que tem de material disponível e não deixar faltar, fazer o pedido logo em seguida. (E5)

É sazonal a questão de material, a gente pede, está sempre pedindo, até nos últimos meses tem vindo material de curativo. Não falta teste rápido, não falta material de citopatológico, mas tipo, eu mantenho um estoque, que não se pode ter, mas é o único jeito. Mas, por exemplo, o copo descartável para a população tomar água, faz oito meses que não vem. (E3)

Faltam vários insumos, desde a parte nossa da Enfermagem, até materiais administrativos. E quando a gente precisa montar um grupo, é tudo com a gente, a gente que fornece. (E7)

Muitas coisas faltam, se a gente quer fazer alguma coisa diferente, não tem. Quer fazer um grupo, um painel, algo diferente, tudo a gente tem que comprar, pagar com o nosso dinheiro. Tu vais ofertar alguma coisa 
Gerenciamento do cuidado em estratégias saúde da família na percepção de enfermeiros 6

diferente em um grupo, que é uma coisa que o pessoal adora, tem que ser patrocinado por nós. (E4)

Percebe-se nos depoimentos dos enfermeiros que a grande dificuldade está na falta de recursos materiais, mesmo quando solicitados à gestão municipal. Segundo os profissionais, medidas como a organização de um estoque na unidade, a aquisição de utensílios para realização de grupos com patrocínio próprio e a realização de pedidos, mesmo não havendo o suprimento, foram adotadas para que não faltassem recursos e a assistência não fosse comprometida.

Quanto aos recursos físicos, aqui salientada a estrutura da unidade, emergiram os seguintes depoimentos, que ilustram uma dicotomia entre os serviços, visto que há locais sem ter estrutura adequada e locais com reformas e adequações que facilitam o desempenho das funções:

Cuido muito pela unidade não ser uma estrutura adequada, não estar dentro das regras, dos padrões, para que cada gestor que entre, esteja ciente da situação. (E4)

Fisicamente melhorou muito nos últimos anos, foram feitos muitos projetos, a gente tem ar condicionado, tem computador e prontuário eletrônico. Então foram ganhos e avanços bem importantes que a gente teve nos últimos três anos [...] a unidade aqui foi reformada, também foi um avanço bem grande que facilitou bastante. (E2)

A realidade dos municípios é marcada por unidades de saúde com diferentes conformações, visualiza-se a adaptação de alguns estabelecimentos com infraestrutura inadequada, a fim de manter o serviço em funcionamento. Tais conformações acontecem visando uma adaptação à saúde da população adscrita na comunidade onde a ESF está inserida, ou até mesmo de acordo com as características populacionais do município. Em contraponto, os enfermeiros perceberam avanços na qualificação da estrutura de novas unidades, possivelmente, pelo acesso a recursos financeiros e também pela informatização das unidades, por meio da implantação do prontuário eletrônico. 
Destaca-se que os enfermeiros entrevistados reconhecem a importância de conhecerem sua equipe, a fim de direcionar melhor o trabalho a ser realizado, de acordo com a aptidão de cada profissional, favorecendo uma assistência de qualidade. Identifica-se, que uma importante estratégia utilizada para o gerenciamento do cuidado é o trabalho em equipe, muito embora haja dificuldade para sua efetivação.

É tu conheceres todos os membros da tua equipe e tentar resgatar o que eles têm de melhor, porque cada pessoa da equipe tem um potencial diferente. Então de início tu vais observar para tentar conhecer a equipe e com o tempo enquadrar e colocar aquele membro numa posição que ele renda bastante e que favoreça o trabalho. (E5)

Conhecer o técnico que trabalha contigo, ver as características do perfil dele e direcionar o tipo de atuação dele. Todos aderem àquela forma de atender, de trabalhar [...]. É muito difícil trabalhar em equipe, porque tem que ser ouvido e ouvir também. (E6)

O trabalho em equipe preconizado pelo modelo de atenção da ESF visa estabelecer relações horizontais entre os profissionais de saúde e ainda lapidar as competências e habilidades desenvolvidas nesse contexto. Os enfermeiros, na gerência, buscam conhecer sua equipe, o perfil dos profissionais, e, a partir disso, elaboram uma melhor distribuição das atribuições, o que contribui nos resultados alcançados pela equipe de saúde.

Emergiu dos depoimentos o posicionamento de que o gerenciamento é um desafio para o enfermeiro, visto a responsabilidade e a complexidade da atividade. Existem múltiplas para que seja concretizado o gerenciamento do cuidado.

Todo o trabalho realizado [...] algo de muita responsabilidade, de bastante complexidade [...] O gerenciamento é o grande desafio para o enfermeiro, porque a nossa prática de técnicas é tranquila, mas os desafios de gerenciamento de personalidades diferentes, de cenários diferentes, eu acho que é o que mais nos desafia e vai desafiar sempre, independente da área que a gente for atuar. (E1) 
Gerenciamento do cuidado em estratégias saúde da família na percepção de enfermeiros 8

O gerenciamento do cuidado exige planejamento, organização, atenção e que a equipe esteja coesa em determinados tipos de atuação. Tu detectas algumas coisas do serviço, como o atual perfil da comunidade, como diabéticos, hipertensos, curativos [...] Primeiro tu analisas a característica da população que tu vais atender e ver o que tu tens disponível dos profissionais que estão na equipe. ( E6)

$\mathrm{Na}$ percepção dos enfermeiros, o gerenciamento se constrói ao reconhecer as especificidades da equipe de saúde e do território, ou seja, o que se modifica de acordo com as vulnerabilidades existentes em cada cenário. Além disso, é imprescindível estar próximo aos usuários adstritos e ter conhecimento do perfil de morbimortalidade dessa comunidade, bem como os aspectos sociais, econômicos, culturais, entre outros, que podem influenciar no processo de saúde-doença.

Com os depoimentos, evidencia-se que o gerenciamento do cuidado está atrelado ao bom funcionamento da unidade ao que se refere ao equilíbrio na disposição de consultas médicas e de enfermagem e, também, à oferta de vacinas, sendo essa logística organizada pela participante após a avaliação situacional.

Então a parte de gerenciamento da equipe, gerenciamento da unidade, como a unidade está andando, o que eu preciso mudar no fluxo de atendimento médico, observando o que está dando certo ou não. Como que está a agenda, se está sendo efetiva, o que há mais necessidade de disponibilizar, equilibrar as consultas de enfermagem, a oferta de vacinas, enfim, de tudo, de todo o trabalho que é realizado por todos da equipe, não só do enfermeiro. (E4)

O organograma da ESF perpassa o planejamento em conjunto da equipe de saúde. Percebe-se que a mediação realizada pelo enfermeiro visa melhorias nos fluxos e nas ofertas de atendimentos, viabilizam adesão aos serviços da ESF e também, melhoram a cobertura dos usuários da sua área adscrita que procura atendimento na unidade. Isso fortalece a importância da dimensão gerencial do enfermeiro, contribuindo para efetivação do gerenciamento do cuidado. 
9 | Bica MC, Cremonese L, Barreto CN, Rodrigues ALM, Alves FQ

Ainda, o gerenciamento do enfermeiro está ligado aos programas do Ministério da Saúde, como Sistema de Informação do Câncer do Colo do Útero (SISCOLO), Sistema de Vigilância Alimentar e Nutricional (SISVAN), Hipertensão Arterial e/ou Diabetes Mellitus (HIPERDIA) e Programa Saúde na Escola (PSE). Esse profissional é o principal protagonista na realização das atividades e atribuições para o andamento do serviço, o qual está relacionado ao correto funcionamento dos programas.

O gerenciamento do enfermeiro está muito centrado nos programas em si, se o enfermeiro está ao lado dos programas, basicamente anda, vamos dizer assim, o enfermeiro é o principal gerenciador nesse sentido, quem vai manter o PSE, o SISCOLO, SISVAN, hiperdia [...]. O enfermeiro não é um coordenador administrativo, não é um gerenciador administrativo, mas acaba sobrando para o enfermeiro essa parte, quando não tem coordenador. (E2)

Observa-se que em algumas unidades de saúde, quando não há o gerente administrativo, há sobrecarga de trabalho no enfermeiro, pois assume essas funções intrinsecamente. Das sete unidades onde foram realizadas as entrevistas, apenas duas contam com o profissional gerente administrativo, as demais apenas com auxiliares administrativos. Outra questão destacada é o grande número de programas a serem retroalimentados, que em sua maioria, são realizados pelo enfermeiro. Porém, é essencial a corresponsabilidade de toda a equipe de saúde nas ações de caráter burocrático e alimentação de sistemas informatizados de saúde.

\section{Discussão}

Ressalta-se que o estoque de produtos e a aquisição de recursos com patrocínio próprio, realizados por alguns enfermeiros, embora sejam compreendidos como positivos, demonstram fragilidade na organização e planejamento das diferentes esferas que compõem a gestão da saúde. Nessa direção, a integralidade do cuidado é fragilizada, o que compromete o atendimento aos usuários, uma vez que pode ocorrer descontinuidade no abastecimento. A situação denota a 
necessidade de um canal de comunicação e participação ativa dos enfermeiros no planejamento do ressuprimento de insumos, uma vez que compreendem as necessidades e a demanda necessária, para, conjuntamente com a gestão municipal, auxiliarem na garantia dos recursos indispensáveis para continuidade do cuidado.

A morosidade da gestão pública resulta na má utilização de recursos físicos e humanos, interferindo diretamente nas ações do sistema de saúde e no exercício dos profissionais da atenção básica. No âmbito da ESF, para desempenho de qualidade, a equipe deve ser organizada, buscando o diagnóstico das necessidades de saúde, elaborando e implementando um plano estratégico de ações, com vistas à resolutividade dos impasses identificados, monitorando e avaliando continuadamente os resultados. ${ }^{9}$

O enfermeiro, por assumir o gerenciamento das unidades de atendimento e coordenar a atividade assistencial, tem papel preponderante no que diz respeito à determinação do material necessário à consecução da assistência. Isso se aplica em aspectos quantitativos e qualitativos, nas definições das especificações técnicas, análise da qualidade, participação do processo de compra e estabelecimento de controle e avaliação. ${ }^{10-12}$

Torna-se necessário que os enfermeiros se visualizem como protagonistas das estratégias e ações das atividades individuais e coletivas, objetivando maior visibilidade à profissão e melhores práticas à comunidade. Esse protagonismo permite elaborar um modelo de gestão do cuidado baseado nas realidades locais visando a reorganização das atividades voltadas à qualidade na atenção à saúde. ${ }^{13-14}$

O conceito de recursos físicos engloba as áreas internas e externas que compõem um serviço de saúde. Uma unidade, especificamente, compreende o espaço físico determinado e especializado para o desenvolvimento de atividade assistenciais, caracterizado por dimensões e instalações diferenciadas. A dimensão refere-se ao tamanho da unidade em função dos equipamentos, da população a ser atendida e das atividades a serem realizadas. ${ }^{12}$ 
Ao encontro disso, ressalta-se a Política Nacional de Humanização que, por meio de suas diretrizes, reforça a importância da ambiência para qualificação da gestão e dos processos de trabalho desenvolvidos nas unidades de saúde. A ambiência compreende a criação de espaços saudáveis que possibilitem a discussão compartilhada, levando em considerações as especificidades do ambiente e dos usuários, para que sejam oportunizados espaços de encontros entre usuários e profissionais de saúde. ${ }^{15}$

O gerenciamento de recursos físicos e ambientais em enfermagem consiste na participação do enfermeiro no planejamento e na alocação desses recursos. O objetivo é de organizar ou gerir, cotidianamente, uma unidade de saúde, provendo segurança, conforto e privacidade aos pacientes e assegurando condições de trabalho apropriadas. ${ }^{12}$

Para a construção de ambientação de Estabelecimento de Assistência à Saúde (EAS), é indispensável atender aos requisitos estabelecidos pelas leis municipais e estaduais, pelo Ministério da Saúde e por outros órgãos governamentais, como a Agência Nacional de Vigilância Sanitária (ANVISA). Esse órgão é regulamentador do sistema de saúde e desempenha ação fiscalizadora quanto à adequação das condições do ambiente onde se processa a atividade e quanto à existência de instalações e equipamentos indispensáveis e condizentes com suas finalidades, com base no controle dos riscos associados. ${ }^{12}$

Ser líder é influenciar os integrantes de sua equipe no decurso das ações, atuando como facilitador e motivador do trabalho. Reforça-se, desse modo, que a dinâmica de liderança de um enfermeiro influencia no processo de trabalho da equipe (agentes comunitários de saúde e técnicos de enfermagem) e, por conseguinte, afeta a qualidade de atenção à saúde prestada aos clientes. Compreende-se que a liderança do enfermeiro no contexto da atenção primária envolve os princípios e diretrizes do SUS, uma vez que denota uma gestão compartilhada, a partir das necessidades de saúde do indivíduo e da coletividade do território onde atua. Além disso, espera-se o reconhecimento do papel da equipe de saúde, considerando o modelo de cuidado 
Gerenciamento do cuidado em estratégias saúde da família na percepção de enfermeiros| 12

preconizado pelas ESF, que visa o usuário e sua família como centro da atenção e uma análise crítica das especificidades do território adstrito. ${ }^{16}$

No que tange ao trabalho em equipe, no campo de práticas da saúde, pensar a união como uma imposição, uma diretriz do sistema, significa um retrocesso sem igual. A construção da harmonia dos profissionais requer interação, comunicação e capacidade para colocar-se no lugar do outro, entendendo os diferentes saberes, em cada especificidade. ${ }^{17-18}$

Articulam-se, na gestão em saúde, estratégias, conhecimentos e recursos institucionais para a intervenção de problemas e necessidades de saúde-doença das comunidades de um território. Elencam-se prioridades e consideram-se ações alternativas para a condução dos processos de trabalho, os quais objetivam a resolução ou o controle dos problemas diagnosticados. A gestão em saúde atua na organização do processo de trabalho, na atuação do profissional de saúde e nas práticas profissionais, visando uma melhor prestação do serviço e a satisfação do usuário. ${ }^{19-20}$

O gerenciamento do cuidado exige planejamento e organização, caracterizando-se como um desafio. A equipe precisa estar preparada para atuar em diferentes determinantes do serviço. Tal fator é imprescindível, pois entende-se que isso viabiliza a oferta adequada de cuidados àquela população, atendendo ao princípio da integralidade.

Torna-se um desafio planejar, executar e avaliar o modelo de gestão do cuidado à saúde adotado. As intervenções necessárias na saúde dependem da construção das relações interpessoais, profissionais, da configuração das redes e de um sistema estruturado, reconhecendo que o modelo de gestão é construído a partir das conexões e vinculações entre os atores envolvidos no processo. ${ }^{13}$

As práticas de gestão do cuidado em saúde vêm sendo delineadas como um novo paradigma na organização da rede de atenção à saúde, sustentadas por um arcabouço teóricocientífico com capacidade de mediar as complexas relações das necessidades demandadas pelos usuários do sistema de saúde. Na horizontalidade do sistema, estrutura-se a rede de atenção 
básica à saúde, que, por meio de um processo de gestão do cuidado qualificado, garante a porta de entrada dos usuários aos diferentes serviços e níveis de complexidade. ${ }^{21-22}$

Para desenvolver a gestão do cuidado integral em enfermagem, no tocante ao gerenciamento, o enfermeiro utiliza ferramentas de gestão como indicadores de saúde, planejamento de materiais e recursos humanos, normas de segurança do paciente, processo de tomada de decisão, entre outras. ${ }^{23} \mathrm{O}$ cuidado prestado diretamente ao paciente engloba técnicas, tecnologias, procedimentos e ações de prevenção, promoção e educação em saúde. As ferramentas que possibilitam o cuidado integral em enfermagem são a sistematização da assistência de enfermagem (SAE), passagem de plantão, acolhimento, consulta de enfermagem, comunicação, visita domiciliária, entre outras. ${ }^{23}$

A capacidade de gerenciar uma equipe de saúde e atender às perspectivas dos usuários requer um profissional equilibrado, que consiga superar as limitações que o serviço apresenta e, além de prestar assistência baseada nos princípios do SUS, saiba lidar com o déficit de pessoal, de materiais, de recursos, bem como com a demanda cada vez maior de usuários. ${ }^{24}$ É fundamental que os gestores das unidades de ESF tenham a capacidade de realizar uma leitura situacional dinâmica e precisa do contexto do sistema de saúde. Dessa forma, há possibilidade de adaptação mais rápida às necessidades sociais e ao direcionamento das ações para a resolutividade do cuidado à saúde e enfermagem da população nos diferentes cenários. ${ }^{14}$

Ao assumir a função gerencial da unidade, em termos práticos, o enfermeiro sofre acúmulo de funções, fator que prejudica o desenvolvimento de seu trabalho e, consequentemente, a eficiência da própria unidade em assistir à população. $O$ excesso de incumbências leva a um atropelamento na execução de ações. ${ }^{24}$

Nas UBS, os enfermeiros desenvolvem, no dia a dia, múltiplas atividades no campo da assistência na ESF, desde a gerência até educação em saúde, ampliando as responsabilidades que, associadas às dificuldades existentes e ao interesse em proporcionar o bom andamento do serviço, 
Gerenciamento do cuidado em estratégias saúde da família na percepção de enfermeiros| 14

sobrecarregam o seu cotidiano, acarretando no sentimento de sobrecarga, estresse e insatisfação com o trabalho. ${ }^{25}$ Desse modo, evidencia-se que o enfermeiro é o principal personagem no gerenciamento do cuidado, sendo que é o responsável pelo manejo da unidade no que tange à assistência e gerência. Ainda percebe-se que as atividades gerenciais sobressaem-se às assistências, sendo que em alguns depoimentos tal fato é atribuído à sobrecarga de trabalho.

Os diferentes níveis de gestão precisam estar engajados no processo de gerenciamento do cuidado, dando meios para que o enfermeiro exerça sua função, uma vez que muitos dos obstáculos encontrados perpassam a atribuição do profissional enfermeiro e alcançam proporções maiores. Tal fato também pode ser atribuído a fatores econômicos e políticos a nível municipal, afinal, é desse a responsabilidade de fornecimento de verbas para aquisição e suprimento de materiais da rede.

Ressalta-se que este estudo apontou as dificuldades encontradas pelos enfermeiros, como a manutenção da continuidade de recursos materiais, sendo os insumos materiais importantes para a logística do cuidado e atendimento à população. Quanto aos recursos humanos, evidenciou-se a preocupação dos enfermeiros em conhecer as potencialidades de suas equipes a fim de direcioná-las à prestação do cuidado, de acordo com suas aptidões. Ainda notou-se a importância do trabalho em equipe, alcançando os objetivos do serviço.

Verifica-se que a formação do enfermeiro está direcionada para uma perspectiva de atendimento pautado na integralidade, o que caracteriza o olhar ampliado na construção de seu perfil profissional, repecurtindo em maior facilidade no gerenciamento das demandas gerenciais e assistenciais. Porém, as competências e habilidades desenvolvidas pelos enfermeiros não anulam o papel dos demais participantes da equipe de se cooresponsabilizarem com essas dimensões, ou seja, no cuidado do indivíduo e de sua família, de acordo com suas necessidades de saúde. Quanto ao conceito de gerenciamento do cuidado, a maioria dos 
participantes fizeram articulações entre gerência e assistência, demonstrando a realização da prática dentro das ESFs.

Menciona-se como limitação a percepção de uma única realidade local, a partir de vulnerabilidades específicas da população adstrita às ESF participantes e ao planejamento da gestão de saúde de um único município. Para investigação dessa especificidade, recomenda-se a realização de futuras pesquisas. Apesar disso, por meio do desenvolvimento deste estudo, foi possível fomentar a reflexão acerca do gerenciamento do cuidado, visando melhorias nas práticas e na qualidade dos cuidados prestados por enfermeiros e equipes de saúde atuantes em ESF.

\section{Conclusão}

Foi possível conhecer as percepções e práticas dos enfermeiros acerca do gerenciamento do cuidado. O trabalho em equipe na ESF, embora reconhecido como essencial para a qualidade do atendimento prestado, possui fragilidades em sua efetivação, pois o enfermeiro assume algumas tarefas que poderiam ser discutidas e compartilhadas com a equipe.

Ainda que as dificuldades permeiem o seu cotidiano, ressaltaram dos depoimentos dos participantes do estudo estratégias para superar tais dificuldades e realizar a prática do gerenciamento do cuidado. Essas estratégias são entendidas pelos enfermeiros como positivas, porém, percebem-se falhas que necessitam ser discutidas junto à gestão municipal, e a inclusão dos profissionais que atuam diferentemente na assistência em saúde no planejamento das ações e serviços ofertados e na aquisição de insumos.

Fica explícita a necessidade dos diferentes níveis de gestão de destinar atenção e investimento para propiciar a prática do enfermeiro em ESF, pois, com a ciência das realidades das ESFs, poderão criar condições adequadas para que os enfermeiros possam trabalhar em consonância com o princípio da integralidade. Ademais, destaca-se a importância do 
Gerenciamento do cuidado em estratégias saúde da família na percepção de enfermeiros| 16

profissional enfermeiro como coordenador ou gerente de uma unidade básica, uma vez que possui habilidades para desenvolver essa atribuição.

\section{Referências}

1. Corrêa VAF, Acioli S, Tinoco TF. Cuidado do enfermeiro na Estratégia Saúde da Família: práticas e fundamentações teóricas. Rev Bras Enferm. 2018;71(Suppl 6):2767-74. doi: 10.1590/0034-7167-2018-0383

2. Treviso P, Peres SC, Silva AD, Santos AA. Competências do enfermeiro na gestão do cuidado. Rev Adm Saúde. 2017;17(69). doi: http://dx.doi.org/10.23973/ras.69.59

3. Mattia BJ, Kleba ME, Prado ML. Formação em enfermagem e a prática profissional: uma revisão integrativa da literatura. Rev Bras Enferm. 2018;71(4):2157-68. doi: http://dx.doi.org/10.1590/0034-71672016-0504

4. Starfield B. Atenção primária: equilíbrio entre as necessidades de saúde, serviços e tecnologia. Brasília (DF): Unesco; 2002.

5. Leal LA, Soares MI, Silva BR, Bernardes A, Camelo SHH. Competências clínicas e gerenciais para enfermeiros hospitalares: visão de egressos de enfermagem. Rev Bras Enferm. 2018;71(Suppl 4):1605-12. doi: http://dx.doi.org/10.1590/0034-7167-2017-0452

6. Silva SS, Assis MMA, Santos AM. Enfermeira como protagonista do gerenciamento do cuidado na estratégia saúde da família: diferentes olhares analisadores. Texto Contexto Enferm. 2017;26(3):e1090016. doi: https://doi.org/10.1590/0104-07072017001090016

7. Minayo MCS. Pesquisa social: teoria, método e criatividade. Rio de Janeiro (RJ): Vozes; 2016.

8. Minayo MCS. Amostragem e saturação em pesquisa qualitativa:consensos e controvérsias. Rev Pesqui Qual [Internet]. 2017 [acesso em 2019 abr 15];5(7):1-12. Disponível em: http://rpq.revista.sepq.org.br/index.php/rpq/article/view/82

9. Arantes LJ, Shimizu HE, Merchán-Hamann E. Contribuições e desafios da Estratégia Saúde da Família na Atenção Primária à Saúde no Brasil: revisão da literatura. Ciênc Saúde Colet. 2016;21(5):1499509. doi: https://doi.org/10.1590/1413-81232015215.19602015

10. Castilho V, Leite MMJ. A administração de recursos materiais na enfermagem. In: Kurcgant P. Administração em enfermagem. São Paulo: EPU; 1991. p.73-88.

11. Bogo PC, Bernardino E, Castilho V, Cruz EDA. O enfermeiro no gerenciamento de materiais em hospitais de ensino. Rev Esc Enferm USP. 2015;49(4):632-9. doi: http://dx.doi.org/10.1590/S0080623420150000400014 
12. Kurcgant P. Gerenciamento em Enfermagem. 3ํe․ São Paulo: Guanabara Koogan; 2016.

13. Sulti ADC, Lima RCD, Freitas PSS, Felsky CN, Galavote HS. O discurso dos gestores da Estratégia Saúde da Família sobre a tomada de decisão na gestão em saúde: desafio para o Sistema Único de Saúde. Saúde Debate. 2015;39(104):172-82. doi: http://dx.doi.org/10.1590/0103-110420151040238

14. Soder R, Oliveira IC, Silva LAA, Santos JLG, Peiter CC, Erdmann AL. Desafios da gestão do cuidado na atenção básica: perspectiva da equipe de enfermagem. Enferm Foco. 2018;9(3):76-80. doi: https://doi.org/10.21675/2357-707X.2018.v9.n3.1496

15. Ministério da Saúde (BR). HumanizaSUS: documento base para gestores trabalhadores do SUS. $4^{\mathbf{a}}$ ed. Brasília (DF): Secretaria de Atenção à Saúde; 2010.

16. Coutinho AF, Medeiros HA, Andrade LDF, Ribeiro LCS. Gestão em enfermagem de pessoal na estratégia saúde da família. Rev Enferm UFPE On Line [Internet]. 2019 [cited 2020 Feb 10];13(1):137-47. Available from: https://periodicos.ufpe.br/revistas/revistaenfermagem/article/view/237019/31190

17. Duarte MLC, Boeck JN. O trabalho em equipe na enfermagem e os limites e possibilidades da estratégia saúde da família. Trab Educ Saúde. 2015;13(3):709-20. doi: http://dx.doi.org/10.1590/1981-7746$\operatorname{sip} 00054$

18. Peruzzo HE, Bega AG, Lopes APAT, Haddad MCFL, Peres AM, Marcon SS. Os desafios de se trabalhar em equipe na estratégia saúde da família. Esc Anna Nery Rev Enferm. 2018;22(4):e20170372. doi: https://doi.org/10.1590/2177-9465-ean-2017-0372

19. Silva MFF, Silva EM, Oliveira SLSS, Abdala GA, Meira MDD. Integralidade na atenção primária à saúde. Rev Fam Ciclos Vida Saúde Contexto Soc [Internet]. 2018 [cited 2019 Aug 16];6(Suppl 1):394-400. Available from: http://www.seer.uftm.edu.br/revistaeletronica/index.php/refacs

20. Signor E, Silva LAA, Gomes IEM, Ribeiro RV, Kessler M, Weiller TH, et al. Educação permanente em saúde: desafios para a gestão em saúde pública. Rev Enferm UFSM. 2015;5(1):01-11. doi: https://doi.org/10.5902/2179769214766

21. Matuda CG, Pinto NRS, Martins CL, Frazão P. Colaboração interprofissional na Estratégia Saúde da Família: implicações para a produção do cuidado e a gestão do trabalho. Ciênc Saúde Colet. 2015;20(8):2511-21. doi: http://dx.doi.org/10.1590/1413-81232015208.11652014

22. Santos JS, Teixeira CF. Política de saúde no Brasil: produção científica 1988-2014. Saúde Debate. 2016;40(108):219-30. doi: http://dx.doi.org/10.1590/0103-1104-20161080018

23. Siewert JS, Rodrigues DB, Malfussi LBH, Andrade SR, Erdmann AL. Gestão do cuidado integral em enfermagem: reflexões sob a perspectiva do pensamento complexo. REME Rev Min Enferm. 2017;21:e1047. doi: 10.5935/1415-2762.20170057 
24. Fernandes JC, Cordeiro BC. O gerenciamento de unidades básicas de saúde no olhar dos enfermeiros gerentes. Rev Enferm UFPE On Line [Internet]. 2018 [cited 2019 Dec 12];12(1):194-202. Available from: https://periodicos.ufpe.br/revistas/revistaenfermagem/article/view/23311/25979

25. Nunes LO, Castanheira ERL, Dias A, Zarili TFT, Sanine PR, Mendonça CS, et al. Importância do gerenciamento local para uma atenção primária à saúde nos moldes de Alma-Ata. Rev Panam Salud Publica. 2018;42:e175. doi: https://doi.org/10.26633/RPSP.2018.175

\section{Autor correspondente}

Matheus Couto Bica

E-mail: matheuscoutobica@gmail.com

Endereço: Rua Saldanha Marinho, 562, Cachoeira do Sul, Rio Grande do Sul, Brasil.

CEP: $96.508-000$

\section{Contribuições de Autoria}

\section{1 - Matheus Couto Bica}

Concepção e planejamento do projeto de pesquisa, obtenção, análise e interpretação dos dados, redação do artigo.

\section{2 - Luiza Cremonese}

Planejamento do projeto de pesquisa, análise, interpretação dos dados, redação e revisão crítica do artigo.

\section{3 - Camila Nunes Barreto}

: Redação e revisão crítica do artigo.

\section{4 - André Luiz Machado Rodrigues}

Redação e revisão crítica do artigo.

\section{5 - Fernanda Quevedo Alves}

Planejamento do projeto de pesquisa, análise, interpretação dos dados, redação e revisão crítica do artigo.

\section{Como citar este artigo}

Bica MC, Cremonese L, Barreto CN, Rodrigues ALM, Alves FQ. Gerenciamento do cuidado em estratégias saúde da família na percepção de enfermeiros. Rev. Enferm. UFSM. 2020 [Acesso em: Anos Mês Dia]; vol.10 e74: P1-18. DOI:https://doi.org/10.5902/2179769242518 